\title{
Jamming in wireless networks under uncertainty
}

\author{
Eitan Altman \\ INRIA Sophia Antipolis, France \\ Email: altman@sophia.inria.fr
}

\author{
Konstantin Avrachenkov \\ INRIA Sophia Antipolis, France \\ Email: k.avrachenkov@sophia.inria.fr
}

\author{
Andrey Garnaev \\ St. Petersburg State University, Russia \\ Email: agarnaev@ rambler.ru
}

\begin{abstract}
The problem of jamming plays an important role in ensuring the quality and security of wireless communications, especially at this moment when wireless networks are quickly becoming ubiquitous. Since jamming can be considered as a game in which jammer is playing against the user (transmitter) who would like to transmit signal with good quality and at the same time with a reasonable amount of energy, game theory is an appropriate tool for dealing with jamming. Here we investigate the effect of partially available information and correlation among sub-carriers on the user behavior. Specifically, to do so we deal with the scenario when the user does not know how jamming efforts are distributed among sub-carriers and the user does not know the fading channels' gains with certainty. As an object function for the user we consider SINR. We consider zerosum games, so all of them can also be viewed as a minimax problem for the user playing against the nature. We study independent fading channel gains scenario as well as dependent fading channel gains scenario, both in discrete and continuous versions. We show that in all the scenarii the jammers equalize the quality of the best sub-carriers for the transmitter on as low level as their power constraints allow. Meanwhile the transmitter distributes his power among these jamming sub-carriers. We find the equilibrium strategies in closed form and specify the range of sub-carriers where the transmitter can expect the jamming attack. Also, we show for independent plot these strategies depend only on the expected value of the transmitters channel gains meanwhile for the dependent plot they depend on the whole spectra of these gains. Thus, for independent plot the behaviour of the jammer is less fine tuned under environment since it works with the expected gains. The user for both scenarios has to take the whole spectra of the jamming gains but, of course, for the independent scenario he is less specific because of the jammer.
\end{abstract}

\section{INTRODUCTION}

The problem of jamming plays an important role in ensuring the quality and security of wireless communications, especially at this moment when wireless networks are quickly becoming ubiquitous. The recent literature covers a variety of jamming problems [1], [2], [4], [5], [6], [13], [14], [15].

Since jamming can be considered as a game in which a jammer is playing against a user (transmitter) who would like to transmit signal with good quality and at the same time with a reasonable amount of energy, game theory is an appropriate tool for dealing with jamming. Here we investigate the effect of partially available information and correlation among subcarriers on the user behavior. Specifically, to do so we deal

\footnotetext{
${ }^{1}$ The work was partly supported by EGIDE ECO-NET grant no.18933SL and RBRG no. 09-01-00334-a
}

with the scenario when the user does not know how jamming efforts are distributed among sub-carriers and the user does not know the fading channels' gains with certainty.

As an object function for the transmitter we consider SINR. To the best of our knowledge, the SINR as an objective function in the power control game was only considered in [3], [10]. In [10] all users have a single common channel and choose between several base stations. And in [3] the authors has considered the power control game between users, not between a user and a jammer. We note that in the regime of low SINR the present objective can serve as an approximation to the Shannon capacity. A central motivation to consider SINR as an objective function and not Shannon capacity, is that current technology for voice over wireless does not try to achieve Shannon capacity but rather uses given codecs that can adapt the transmission rate to the SINR; these turn out to adapt the rate in a way that is linear in the SINR over a wide range of throughput. The SINR has therefore been used very often to represent directly the throughput see [11], [12]. The validity of this can be seen e.g. in [9, p. 151, 222, 239]. As we see from [9, Fig. 10.4, p. 222], the ratio between the throughput and the SINR is close to a constant throughout long range of bit rates. For example, between 16Kbps and $256 \mathrm{Kbps}$, the maximum variation around the median value is less than $20 \%$.

We analyse jamming as a zero-sum game. Thus, it can also be viewed as a minimax problem for the user playing against the nature. We study independent fading channel gains scenario as well as dependent fading channel gains scenario, both in discrete and continuous versions. Independent and dependent channel gains scenarios can be considered as scenarios where transmitter has different information about environment. To demonstrate how this information impacts on the transmitter's behaviour we perform numerical modelling employing the obtained closed form solutions. Also, we show that in all the scenarii the jammers equalize the quality of the best sub-carriers for transmitter on as low level as their power constraints allow. Meanwhile the transmitter distributes his power among these jamming sub-carriers. We find the equilibrium strategies in a closed form and specify the range of sub-carriers where the transmitter can expect the jamming attack. Using this formulas we show for independent plot these strategies depend only on the expected value of the transmitters channel gains meanwhile for the dependent plot they depend 
on the whole spectra of these gains. Thus, for independent plot the behaviour of the jammer is less fine tuned under environment since it works with the expected gains. The user for both scenarios has to take the whole spectra of the jamming gains but, of course, for the independent scenario he is less specific because of the jammer.

It is worth to mention that the considered jamming game relates to resource allocation games which have a lot of application with military favour (say, Colonel Blotto game [8] or Star War game [7]) and in search theory [7].

\section{Formulation of the Game}

The transmitter should assign different power levels for different sub-carriers to maximize the SINR meanwhile the other user (jammer) wants to minimize this objective function. This game can also be viewed as a minimax problem for the transmitter against the nature. The strategy of the transmitter is $T=\left(T_{1}, \ldots, T_{n}\right)$ with $T_{i} \geq 0, i \in[1, n]$ such that $\sum_{i=1}^{n} T_{i}=\bar{T}$, where $\bar{T}>0$ is the total power available to the transmitter, $n$ is the number of sub-carriers and $T_{i}$ is the power level assigned to each sub-carrier $i$. The strategy of the jammer is $J=\left(J_{1}, \ldots, J_{n}\right)$ with $J_{i} \geq 0, i \in[1, n]$ such that $\sum_{i=1}^{n} J_{i}=\bar{J}$, where $\bar{J}>0$ is the total power available to the jammer.

We assume that the transmitter does not know the fading channels' gains with certainty, namely, the fading channel gains can be random, i.e. with probability $p^{k}, k \in[1, K]$ they are $\alpha_{i}^{k}, \beta_{i}^{k}, i \in[1, n]$. The transmitter payoff is given as his average SINR:

$$
v(T, J)=\sum_{k=1}^{K} p^{k} \sum_{i=1}^{n} \frac{\alpha_{i}^{k} T_{i}}{N^{0 k}+\beta_{i}^{k} J_{i}} .
$$

We assume that players know that all the possible variations of fading channel gains $\alpha_{i}^{k}$, $\beta_{i}^{k}$, the noise levels $N^{0 k}$ and the probabilities $p^{k}$. The total powers $\bar{T}$ and $\bar{J}$ are also known to both players. In the situation when the transmitter and the jammer are both active players, the payoff to the jammer is $-v(T, J)$. That is, we consider a zero-sum game. We would like to emphasize that the formulation (1) allows one to study the correlation among different sub-carriers.

Also we consider a particular important case when gains for both users are independent. In such a case the transmitter payoff is given as follows:

$$
v(T, J)=\sum_{k=1}^{K} \sum_{l=1}^{L} p^{k} q^{l} \sum_{i=1}^{n} \frac{\alpha_{i}^{l} T_{i}}{N^{0}+\beta_{i}^{k} J_{i}},
$$

where $p^{k}$ is the probability that the fading channel gain $\beta_{i}$ is in the state $k$ (so, it is equal to $\beta_{i}^{k}$ ), and $q^{l}$ is the probability that the fading channel gain $\alpha_{i}$ is in the state $l$ (so, it is equal to $\alpha_{i}^{l}$ ) and $N^{0}$ is the background noise level in all the sub-carriers, which we assume to be constant. Besides these discrete models we will also study their continuous versions with the continuous distributions for the fading channels' gains. The formulation and the analysis of the continuous models we will discuss in a separate section.

\section{OPTIMIZATION FORMULATIONS FOR THE DEPENDENT FADING CHANNEL GAIN SCENARIO}

Let us consider the situation for the dependent fading channel gain scenario when one of the players is inactive. Thus, in this section we deal with the optimization problems. First we consider the case when the jammer is inactive, so his strategy $J=\left(J_{1}, \ldots, J_{n}\right)$ is fixed and is known to the transmitter. Since (1) is linear in $T$, the optimal strategy for the transmitter for a fixed jammer strategy can be found as given in the next theorem.

Theorem 1: Let the jammer be an inactive player, so $J$ is fixed. Then the optimal strategy for the transmitter is to transmit the signal within the sub-carriers with the maximal induced expected fading gains $\sum_{k=1}^{K} p_{k} \alpha_{i}^{k} /\left(N^{0 k}+\beta_{i}^{k} J_{i}\right)$.

Next let us assume that the transmitter fixes his strategy, so, transmitter is an inactive player and he would like to know which payoff he can get under the most unfavorable circumstances, namely, the transmitter wants to find the minimum of $v_{T}(J):=v(T, J)$ for a fixed $T$. Then, we have the following result.

Theorem 2: Let the transmitter be an inactive player, so $T=\left(T_{1}, \ldots, T_{n}\right)$ is fixed. Then the worst payoff the transmitter could have is

$$
v=\sum_{k=1}^{K} p^{k} \sum_{i=1}^{n} \frac{\alpha_{i}^{k} T_{i}}{N^{0 k}+\beta_{i}^{k} J_{i}^{o}(\nu)},
$$

where $\left(J_{1}^{o}(\nu), \ldots, J_{n}^{o}(\nu)\right.$ is the optimal jamming strategy and (a) if

$$
T_{i} \sum_{k=1}^{K} \frac{\alpha_{i}^{k} \beta_{i}^{k} p^{k}}{\left(N^{0 k}\right)^{2}}>\nu
$$

then $J_{i}^{o}(\nu)$ is the unique root of the following equation

$$
F_{i}(x)=\nu,
$$

where

$$
F_{i}(x)=T_{i} \sum_{k=1}^{K} \frac{\alpha_{i}^{k} \beta_{i}^{k} p^{k}}{\left(N^{0 k}+\beta_{i}^{k} x\right)^{2}},
$$

and $\nu$ is the unique root of the equation

$$
\sum_{i=1}^{n} J_{i}^{o}(\nu)=\bar{J}
$$

(b) if

$$
T_{i} \sum_{k=1}^{K} \frac{\alpha_{i}^{k} \beta_{i}^{k} p^{k}}{\left(N^{0 k}\right)^{2}} \leq \nu
$$

then $J_{i}^{o}(\nu)=0$. 
Proof: Since

$$
F_{i}\left(T_{i}\right)=\frac{\partial v}{\partial J_{i}}
$$

and

$$
\frac{\partial^{2} v}{\partial J_{i}^{2}}=T_{i} \sum_{k=1}^{K} \frac{2 \alpha_{i}^{k}\left(\beta_{i}^{k}\right)^{2} p^{k}}{\left(N^{0 k}+\beta_{i}^{k} J_{i}\right)^{3}},
$$

by Kuhn-Tucker Theorem, there is a $\nu$ (Lagrange multiplier) such that

$$
F_{i}\left(T_{i}\right) \begin{cases}=\nu, & \text { if } J_{i}>0, \\ \leq \nu, & \text { if } J_{i}=0 .\end{cases}
$$

Note that $J_{i}^{o}(\nu)$ is correctly defined by (5) since $F_{i}(x)$ is decreasing in $x$ and

$$
F_{i}(0)=T_{i} \sum_{k=1}^{K} \frac{\alpha_{i}^{k} \beta_{i}^{k} p^{k}}{\left(N^{0 k}\right)^{2}} .
$$

Besides, $G(\nu):=\sum_{i=1}^{n} J_{i}^{o}(\nu)$ is non-negative, continuous for $\nu>0$ and also decreasing in $\nu$ while it is positive, and $G(0+)=\infty, G(\nu)=0$ for $\nu \geq \max _{i} F_{i}(0)$. So, there is the unique root of (3). This completes the proof of Theorem 2.

\section{ZERO-SUM POWER GAME UNDER UNCERTAINTY}

In the situation when both the transmitter and the jammer are active players we are in the context of the zero-sum game and the payoff to the jammer is $-v(T, J)$. We will look for an equilibrium and the value of the game, that is, we want to find the strategies $\left(T^{*}, J^{*}\right) \in A \times B$ such that

$$
v\left(T, J^{*}\right) \leq v\left(T^{*}, J^{*}\right) \leq v\left(T^{*}, J\right) \text { for any }(T, J) \in A \times B,
$$

where $A$ and $B$ are the sets of all the strategies of the transmitter and the jammer, respectively, and $v=v\left(T^{*}, J^{*}\right)$ is the value of the game. We have the following result describing the equilibrium of the game for the case of the dependent fading channel gains.

Theorem 3: The equilibrium of the game with the dependent fading channel gains is $(T, J)=(T(\omega), J(\omega))$ where

$$
J_{i}(\omega)= \begin{cases}\text { the unique root of } R_{i}(x)=\omega, & \sum_{k=1}^{K} \frac{p^{k} \alpha_{i}^{k}}{N^{0 k}}>\omega, \\ 0, & \sum_{k=1}^{K} \frac{p^{k} \alpha_{i}^{k}}{N^{0 k}} \leq \omega\end{cases}
$$

with

$$
R_{i}(x):=\sum_{k=1}^{K} \frac{\alpha_{i}^{k} p_{k}}{N^{0 k}+\beta_{i}^{k} x}
$$

and $\omega=\omega_{*}$ is the unique positive root of the equation

$$
H_{J}(\omega):=\sum_{i=1}^{n} J_{i}(\omega)=\bar{J} .
$$

Also,

$$
T_{i}(\omega)= \begin{cases}\bar{T} \frac{\sum_{k=1}^{K} \frac{\alpha_{i}^{k} \beta_{i}^{k} p^{k}}{\left(N^{0 k}+\beta_{i}^{k} J_{i}(\omega)\right)^{2}}}{\sum_{i \in I^{J}(\omega)} \sum_{k=1}^{K} \frac{\alpha_{i}^{k} \beta_{i}^{k} p^{k}}{\left(N^{0 k}+\beta_{i}^{k} J_{i}(\omega)\right)^{2}}} & i \in I^{J}(\omega), \\ 0, & i \notin I^{J}(\omega),\end{cases}
$$

with

$$
I^{J}(\omega)=\left\{i \in[1, n]: J_{i}(\omega)>0\right\} .
$$

And, $v=\omega \bar{T}$ is the value of the game.

Proof: Since the payoff (1) is linear in $T$ and concave in $J$, $(T, J)$ is the equilibrium if and only if there are positive $\omega$ such that $\nu$ (Lagrange multiplier) such that

(a) if

$$
T_{i} \sum_{k=1}^{K} \frac{\alpha_{i}^{k} \beta_{i}^{k} p^{k}}{\left(N^{0 k}\right)^{2}}>\nu
$$

then $J_{i}$ is the unique root of the equation

$$
T_{i} \sum_{k=1}^{K} \frac{\alpha_{i}^{k} \beta_{i}^{k} p^{k}}{\left(N^{0 k}+\beta_{i}^{k} J_{i}\right)^{2}}=\nu
$$

(b) if

$$
T_{i} \sum_{k=1}^{K} \frac{\alpha_{i}^{k} \beta_{i}^{k} p^{k}}{\left(N^{0 k}\right)^{2}} \leq \nu
$$

then

$$
J_{i}=0,
$$

(c) the following relation holds:

$$
T_{i}\left\{\begin{array}{l}
\geq 0, \quad \text { if } \sum_{k=1}^{K} \frac{p^{k} \alpha_{i}^{k}}{N^{0 k}+\beta_{i}^{k} J_{i}}=\omega, \\
=0, \quad \text { if } \sum_{k=1}^{K} \frac{p^{k} \alpha_{i}^{k}}{N^{0 k}+\beta_{i}^{k} J_{i}}<\omega .
\end{array}\right.
$$

By (a) and (b), $J_{i}=0$ for $T_{i}=0$. Since we deal with zerosum game it is reasonable to look for the jammer's optimal strategy among such strategies hampering only the sub-carriers employed by the transmitter, namely, $T_{i}=0$ for $J_{i}=0$. Since $R_{i}(x)$ is decreasing and continuous in $x$, it yields, by (10), that the optimal $J_{i}$ has to be of the form (5), where $\omega$ is the unique positive root of (6). Then, it is clear that $T_{i}(\omega)$ given by (7) turns the system of the equations (8) into equalities with

$$
\nu=\frac{\bar{T}}{\sum_{i \in I^{J}(\omega)} \sum_{k=1}^{K} \frac{\alpha_{i}^{k} \beta_{i}^{k} p^{k}}{\left(N^{0 k}+\beta_{i}^{k} J_{i}(\omega)\right)^{2}} .}
$$

This completes the proof of Theorem 3.

It is interesting that the optimal jamming strategy assumes to jam as much channels as possible with the best expected SINR meanwhile transmitter just adjusts to the situation making 
the best average harmed channels equally attractive for the jammer.

\section{A PARTICULAR CASE: THE FADING COEFFICIENT GAINS ARE KNOWN TO THE PLAYERS}

We will assume that all the fading channel gains $\alpha_{i}$ and $\beta_{i}$, the noise levels $N^{0}$, the total powers $\bar{T}$ and $\bar{J}$ are known to both players. Then the object function (payoff) to transmitter is as folows:

$$
v(T, J)=\sum_{i=1}^{n} \frac{\alpha_{i} T_{i}}{N^{0}+\beta_{i} J_{i}}
$$

Since (11) is linear in $T$, the optimal strategy of the transmitter for a fixed jammer strategy (so, jammer is an unactive player) can be found as given in the next statement.

Theorem 4: Let the jammer be an inactive player, so $J$ is fixed. Then the the optimal strategy for the transmitter is to transmit the signal by the sub-carriers with the maximal induced fading gains $\alpha_{i} /\left(N^{0}+\beta_{i} J_{i}\right)$.

Let the transmitter be an inactive player (so, his strategy is fixed) and he would like to know which payoff he can get under the most unfavorable circumstances, namely transmitter wants to find the minimum of $v_{T}(J):=v(T, J)$ for a fixed $T$. Then, the optimal strategy for the jammer has a water-filling form.

Theorem 5: Let the transmitter be an inactive player, so $T$ is fixed. Then the worst payoff he could have is $v=$ $\sum_{i=1}^{n}\left(\alpha_{i} T_{i} /\left(N^{0}+\beta_{i} J_{i}^{o}(\omega)\right)\right)$, where $J_{i}^{o}(\omega)$ is the optimal jamming strategy:

$$
J_{i}^{o}(\omega)=\frac{1}{\beta_{i}}\left[\sqrt{\frac{T_{i} \alpha_{i} \beta_{i}}{\omega}}-N_{0}\right]_{+}, \quad i \in[1, n]
$$

and $\omega$ is the unique root of the water filling equation

$$
\frac{1}{\beta_{i}}\left[\sqrt{\frac{T_{i} \alpha_{i} \beta_{i}}{\omega}}-N_{0}\right]_{+}=\bar{J} .
$$

In the situation when the transmitter and the jammer are active players we have the following result supplying the equilibrium strategies in a closed form and specifying the range of sub-carriers where the transmitter can expect the attack of the jammer.

Theorem 6: In the zero-sum game formulation, $(T, J)=$ $(T(\omega), J(\omega))$ is the equilibrium where

$$
\begin{aligned}
& J_{i}(\omega)=\frac{\alpha_{i}}{\beta_{i}}\left[\frac{1}{\omega}-\frac{N^{0}}{\alpha_{i}}\right]_{+}, \quad i \in[1, n], \\
& T_{i}(\omega)= \begin{cases}\frac{\bar{T} \alpha_{i} / \beta_{i}}{\sum_{k \in I^{J}(\omega)}\left(\alpha_{k} / \beta_{k}\right)}, & i \in I^{J}(\omega), \\
0, & i \notin I^{J}(\omega),\end{cases}
\end{aligned}
$$

where

$$
I^{J}(\omega)=\left\{i \in[1, n]: J_{i}(\omega)>0\right\},
$$

and $\omega$ is the unique root of the following water-filling equation:

$$
\sum_{i=1}^{n} \frac{\alpha_{i}}{\beta_{i}}\left[\frac{1}{\omega}-\frac{N^{0}}{\alpha_{i}}\right]_{+}=\bar{J}
$$

And, $v=\omega \bar{T}$ is the value of the game.

It is interesting that the optimal jamming strategy has water filling form which depends on the transmitter fading channel gains $\left(\alpha_{i}\right)$ and on the relation between the transmitter and jamming fading channel gains $\left(\alpha_{i} / \beta_{i}\right)$ where the transmitter fading channel gains plays bigger role since it defines the sequence of the channels harmed by jammer. The level of the power the jammer has defines the number of the best channels he can harm. Transmitter just adjusts to the situation making the best harmed channel equally attractive for the jammer.

VI. A PARTICULAR CASE: ZERO-SUM GAME FOR THE FADING CHANNEL GAINS INDEPENDENT WITH RESPECT TO THE OPPONENT

In the case of the fading channel gains independent with respect to the opponent the payoff (2) is equivalent to

$$
v(T, J)=\sum_{k=1}^{K} p^{k} \sum_{i=1}^{n} \frac{\bar{\alpha}_{i} T_{i}}{N^{0}+\beta_{i}^{k} J_{i}}
$$

where $\bar{\alpha}_{i}=\sum_{l=1}^{L} q^{l} \alpha_{i}^{l}$ is the expected fading channel gain for the transmitter. Then we have the following result describing the equilibrium of the game.

Theorem 7: The equilibrium of the game for the independent fading channel gain plot is $(T, J)=(T(\omega), J(\omega))$ where

$$
J_{i}(\omega)= \begin{cases}\text { the unique root of } R_{i}(x)=\omega, & \text { if } \frac{\bar{\alpha}_{i}}{N^{0}}>\omega, \\ 0, & \text { if } \frac{\alpha_{i}}{N^{0}} \leq \omega\end{cases}
$$

with

$$
R_{i}(x):=\bar{\alpha}_{i} \sum_{k=1}^{K} \frac{p_{k}}{N^{0}+\beta_{i}^{k} x}
$$

and $\omega=\omega_{*}$ is the unique positive root of the equation

$$
H_{J}(\omega):=\sum_{i=1}^{n} J_{i}(\omega)=\bar{J} .
$$

Also,

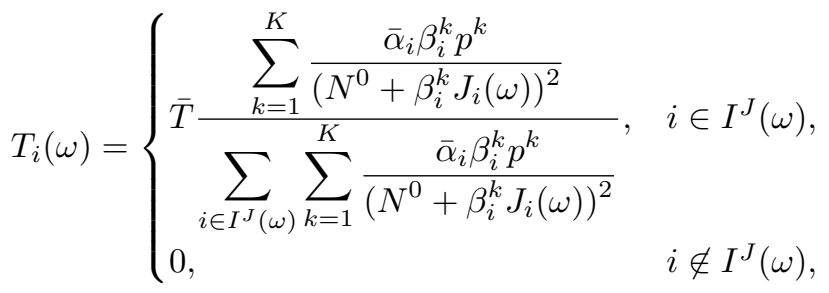


with

$$
I^{J}(\omega)=\left\{i \in[1, n]: J_{i}(\omega)>0\right\} .
$$

Also, $v=\omega \bar{T}$ is the value of the game.

\section{NUMERICAL EXAMPLES: COMPARING OPTIMIZATION AND GAME PLOTS}

To compare the optimization and game-theoretical approaches we consider a system consisting of $n=5$ subcarriers.

First we consider the situation when the transmitter knows the fading channel gains. Assume that the background noise is $N^{0}=1$. Let the transmitter and the jammer have the same power budgets, namely, let $\bar{T}=\bar{J}=3$. The jammer fading gains are given by the linear law $\beta_{i}=i, i \in[1,5]$. The transmitter fading gains are $\alpha_{i}=6-i, i \in[2,5]$ and $\alpha_{1}=5,10,20$. For the optimization scenario we assume that transmitter applies the uniform strategy $T^{0}$, so $T^{0}=$ $(3 / 5,3 / 5,3 / 5,3 / 5,3 / 5)$. Then, in Table 1 the optimal strategies $(T, J)$ for game-theoretical plot and $J^{0}$ for optimization plot and corresponding payoffs are given. For the game plot while the quality of the first sub-carriers (relation of $\alpha_{1} / N^{0}$ ) is increasing the players more and more concentrate their efforts on this sub-carrier till they completely switch their efforts just for this sub-carrier. In the optimization approach the influence of this sub-carrier is increasing but not so big as in the game plot. The ratio of game and optimization payoffs increases from 1.31 for $\alpha_{1}=5$ to 2.04 for $\alpha_{1}=20$.

TABLE I

THE GAME-THEORETICAL AND OPTIMIZATION PLOTS

\begin{tabular}{|c|c|c|c|c|c|c|c|}
\hline$\alpha_{1}$ & $v$ & $T / J$ & 1 & 2 & 3 & 4 & 5 \\
\hline 5 & 5.016 & $J$ & 1.990 & 0.696 & 0.265 & 0.049 & 0.000 \\
& & $T$ & 1.765 & 0.706 & 0.353 & 0.176 & 0.000 \\
& 3.826 & $J^{0}$ & 1.035 & 0.787 & 0.577 & 0.394 & 0.207 \\
10 & 8.069 & $J$ & 2.718 & 0.244 & 0.038 & 0.000 & 0.000 \\
& & $T$ & 2.308 & 0.462 & 0.231 & 0.000 & 0.000 \\
& 5.144 & $J^{0}$ & 1.482 & 0.610 & 0.452 & 0.305 & 0.151 \\
20 & 15.000 & $J$ & 3.000 & 0.000 & 0.000 & 0.000 & 0.000 \\
& & $T$ & 3.000 & 0.000 & 0.000 & 0.000 & 0.000 \\
& 7.342 & $J^{0}$ & 1.939 & 0.429 & 0.324 & 0.215 & 0.094 \\
\hline
\end{tabular}

Now consider the situation where the transmitter does not know the fading channel gains and we assume that the system consists from $n=5$ sub-carriers and it can be in two states $(K=2)$ with background noise $N^{0}=(1,1)$. The transmitter and the jammer have the same power budgets, namely, let $\bar{T}=\bar{J}=3$. The jammer fading and transmitter gains are given by $((1,5),(2,4)),(3,3),(4,2),(5,1))$ and $((20,1),(4,2),(3,3),(2,4),(1,5))$, respectively. For the optimization scenario we assume that the transmitter applies the uniform strategy $T^{0}$, so $T^{0}=(3 / 5,3 / 5,3 / 5,3 / 5,3 / 5)$. Then, in Table 2 the optimal strategies $\left(T_{d}, J_{d}\right)$ and $\left(T_{i}, J_{i}\right)$ for dependent and independent game-theoretical plot and $J^{0}$ for optimization plot and corresponding payoffs are given for the probability $p^{1}=0.0(0.2) 1.0$ of being in state 1 . We assume that for independent plot $K=L, N^{0}=1$ and $q^{i}=p^{i}$, $i \in[1, L]$ Of course, for $p^{1}=0$ the strategies in the game and optimization frameworks coincide with the strategies from Table 1 . For $p^{1}=0.2$ in the game framework of the dependent plot the players still stick to the same strategies in spite of the fact that the value of the game goes down meanwhile for the the independent plot these strategies differ from each other. In the optimization framework the jammer tunes his behaviour more flexibly already for $p^{1}=0.2$ which allows him to bring bigger harm. With decreasing $p^{1}$ more and more sub-carriers become involved into strategies and from $p^{1}=0.6$ all the sub-carriers have to be taken into account by players. Figure 1 shows the dependence of the optimal payoff and the value of the game for both plots on $p^{1}$. The value of the game for the independent case is always less than the optimization payoff and the value for the dependent one. Thus, we demonstrate that one can take advantage from the knowledge of the correlation structure. At two boundary points $p^{1}=0$ and $p^{1}=1$ the value of the games coincide, and the maximally differ at $p^{1}=0.6$. For $p_{1} \in[0.3,0.5]$ the optimization payoff for the uniform transmitter strategy is greater than the value of the game for the dependent case.

TABLE II

THE GAME-THEORETICAL AND OPTIMIZATION PLOTS

\begin{tabular}{|c|c|c|c|c|c|c|c|}
\hline$p^{1}$ & $v$ & $T / J$ & 1 & 2 & 3 & 4 & 5 \\
\hline \multirow[t]{5}{*}{0} & 3.826 & $J_{O}$ & 0.207 & 0.394 & 0.577 & 0.787 & 1.035 \\
\hline & 5.016 & $J_{d}$ & 0.000 & 0.049 & 0.265 & 0.696 & 1.990 \\
\hline & & $T_{d}$ & 0.000 & 1.622 & 0.811 & 0.405 & 0.162 \\
\hline & 5.016 & $J_{i}$ & 0.000 & 0.049 & 0.265 & 0.696 & 1.990 \\
\hline & & $T_{i}$ & 0.000 & 1.622 & 0.811 & 0.405 & 0.162 \\
\hline \multirow[t]{5}{*}{0.2} & 5.167 & $J_{o}$ & 0.780 & 0.403 & 0.503 & 0.612 & 0.702 \\
\hline & 5.760 & $J_{d}$ & 1.214 & 0.076 & 0.188 & 0.405 & 1.117 \\
\hline & & $T_{d}$ & 0.213 & 1.177 & 0.875 & 0.518 & 0.217 \\
\hline & 4.638 & $J_{i}$ & 0.609 & 0.157 & 0.313 & 0.583 & 1.338 \\
\hline & & $T_{i}$ & 0.484 & 1.085 & 0.749 & 0.466 & 0.215 \\
\hline \multirow[t]{5}{*}{0.4} & 6.003 & $J_{o}$ & 1.239 & 0.410 & 0.444 & 0.472 & 0.435 \\
\hline & 7.214 & $J_{d}$ & 2.392 & 0.059 & 0.083 & 0.136 & 0.331 \\
\hline & & $T_{d}$ & 0.116 & 0.927 & 0.938 & 0.699 & 0.321 \\
\hline & 5.285 & $J_{i}$ & 1.776 & 0.191 & 0.234 & 0.308 & 0.491 \\
\hline & & $T_{i}$ & 0.192 & 0.897 & 0.837 & 0.674 & 0.401 \\
\hline \multirow[t]{5}{*}{0.6} & 6.582 & $J_{o}$ & 1.559 & 0.421 & 0.400 & 0.366 & 0.253 \\
\hline & 9.123 & $J_{d}$ & 2.979 & 0.021 & 0.000 & 0.000 & 0.000 \\
\hline & & $T_{d}$ & 0.291 & 2.709 & 0.000 & 0.000 & 0.000 \\
\hline & 7.081 & $J_{i}$ & 2.689 & 0.131 & 0.090 & 0.059 & 0.031 \\
\hline & & $T_{i}$ & 0.084 & 0.580 & 0.700 & 0.778 & 0.859 \\
\hline \multirow[t]{5}{*}{0.8} & 7.008 & $J_{O}$ & 1.783 & 0.428 & 0.361 & 0.281 & 0.148 \\
\hline & 12.038 & $J_{d}$ & 3.000 & 0.000 & 0.000 & 0.000 & 0.000 \\
\hline & & $T_{d}$ & 3.000 & 0.000 & 0.000 & 0.000 & 0.000 \\
\hline & 10.373 & $J_{i}$ & 2.983 & 0.017 & 0.000 & 0.000 & 0.000 \\
\hline & & $T_{i}$ & 0.301 & 2.699 & 0.000 & 0.000 & 0.000 \\
\hline \multirow[t]{5}{*}{1} & 7.342 & $J_{o}$ & 1.939 & 0.429 & 0.324 & 0.215 & 0.094 \\
\hline & 15.000 & $J_{d}$ & 3.000 & 0.000 & 0.000 & 0.000 & 0.000 \\
\hline & & $T_{d}$ & 3.000 & 0.000 & 0.000 & 0.000 & 0.000 \\
\hline & 15.000 & $J_{i}$ & 3.000 & 0.000 & 0.000 & 0.000 & 0.000 \\
\hline & & $T_{i}$ & 3.000 & 0.000 & 0.000 & 0.000 & 0.000 \\
\hline
\end{tabular}

\section{CONTINUOUS MODEL}

In this section we assume that the fading channel gains coefficients change in a continuous set and the transmitter 

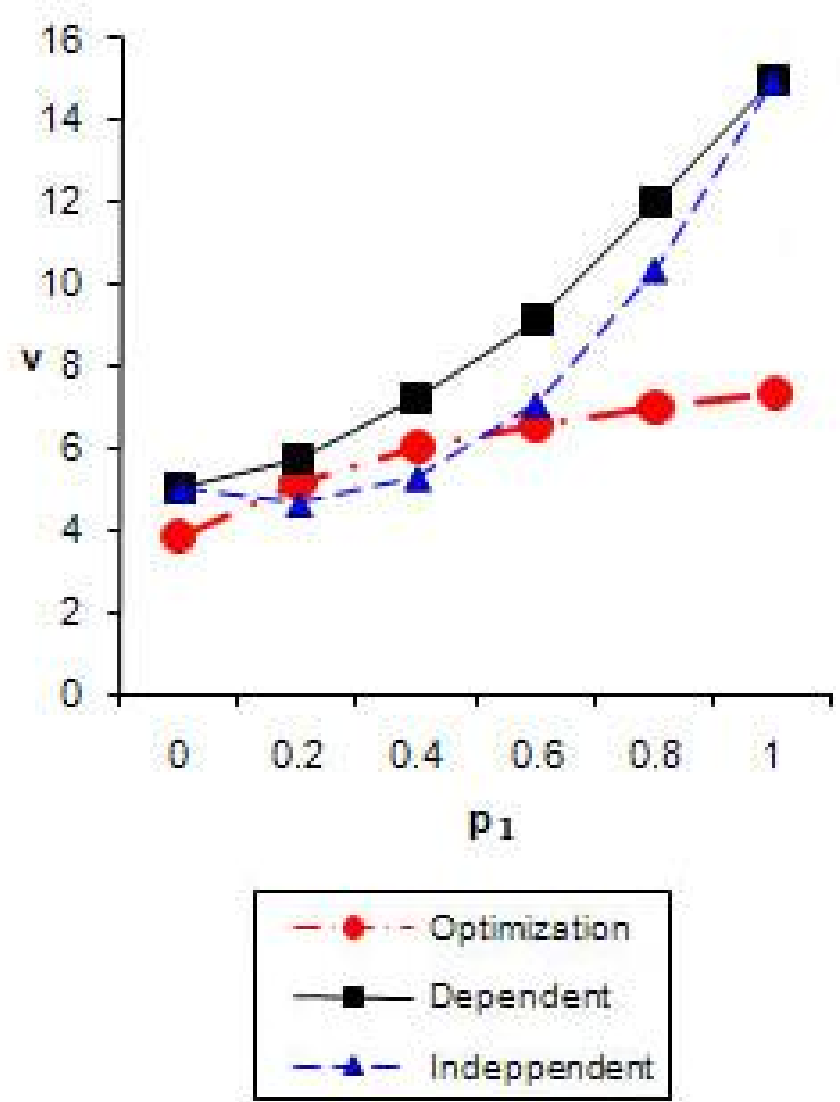

Fig. 1. The optimal payoff and the value of the game

knows the distribution of the fading channel gains. Then the payoff to the transmitter is given as follows:

$$
v(T, J)=\sum_{i=1}^{n} \int_{0}^{\infty} \int_{0}^{\infty} \sigma_{i}(\alpha) \rho_{i}(\beta) \frac{\alpha T_{i}}{N^{0}+\beta J_{i}} d \alpha d \beta .
$$

If $\sigma_{i}(\alpha)$ and $\rho_{i}(\beta)$ are independent of sub-carrier, namely, $\sigma_{i}(\alpha)=\sigma(\alpha)$ and $\rho_{i}(\beta)=\rho(\beta)$ for $i \in[1, n]$ then the optimal strategies for the transmitter and the jammer are the uniform ones. Otherwise the optimal strategy of the jammer is given as follows:

(a) if

$$
R_{i}(0)>\omega
$$

then $J_{i}(\omega)$ is the unique root of

$$
R_{i}(x)=\omega,
$$

(b) if

$$
R_{i}(0) \leq \omega
$$

then

$$
R_{i}(x)=0
$$

where

$$
R_{i}(x):=\int_{0}^{\infty} \int_{0}^{\infty} \frac{\alpha}{N^{0}+\beta x} \sigma_{i}(\alpha) \rho_{i}(\beta) d \alpha d \beta
$$

and $\omega=\omega_{*}$ is the unique positive root of the equation $\sum_{i=1}^{n} J_{i}(\omega)=\bar{J}$. Also, the optimal strategy of the transmitter is given as follows:

(a) for $i \in I^{J}(\omega)$

$$
T_{i}(\omega)=\bar{T} \frac{\int_{0}^{\infty} \int_{0}^{\infty} \frac{\alpha \beta}{\left(N^{0}+\beta J_{i}(\omega)\right)^{2}} \sigma_{i}(\alpha) \rho_{i}(\beta) d \alpha d \beta}{\sum_{i \in I^{J}(\omega)} \int_{0}^{\infty} \int_{0}^{\infty} \frac{\alpha \beta}{\left(N^{0}+\beta J_{i}(\omega)\right)^{2}} \sigma_{i}(\alpha) \rho_{i}(\beta) d \alpha d \beta},
$$

(b) for $i \notin I^{J}(\omega)$

$$
T_{i}(\omega)=0
$$

with

$$
I^{J}(\omega)=\left\{i \in[1, n]: J_{i}(\omega)>0\right\} .
$$

And, $v=\omega \bar{T}$ is the value of the game.

\section{CONCLUSIONS}

In this paper we have studied how the available information and sub-carriers fading channel gains correlation impact the transmitter in the case of jamming. As an object function to the transmitter we have considered SINR. We have considered optimization and zero-sum game plots. The zero-sum game can also be viewed as a minimax problem for the user playing against the nature. We have studied independent fading channel gains scenario as well as dependent fading channel gains scenario. We have shown that in all the scenarii the jammers equalize the quality of the best sub-carriers for the transmitter on as low level as their power constraints allow, meanwhile the transmitter distributes his power among these jamming sub-carriers. We have found the equilibrium strategies in closed form and specified the range of sub-carriers where the transmitter can expect the jamming attack.

An interesting conclusion also can be done from comparing the closed form optimal strategies for the independent and dependent case. Namely, for independent plot these strategies depend only on $\bar{\alpha}_{i}$, so they depend on the expected value of the transmitters channel gains meanwhile for the dependent plot they depend on the whole spectra of these gains. Thus, for independent plot the behaviour of the jammer is less fine tuned under environment since it works with the expected gains. The user for both scenarios has to take the whole spectra of the jamming gains but, of course, for the independent scenario he is less specific because of the jammer.

\section{REFERENCES}

[1] E. Altman, K. Avrachenkov and A. Garnaev, "A jamming game in wireless networks with transmission cost". in Proc. of NET-COOP 2007. Lecture Notes in Computer Science, v.4465, pp.1-12, 2007.

[2] E. Altman, K. Avrachenkov and A. Garnaev, "Fair resource allocation in wireless networks in the presence of a jammer", in Proc. of ValueTools 2008, 2008.

[3] E. Altman, K. Avrachenkov and A. Garnaev, "Transmission power control game with SINR as objective function", in Proc. of NET-COOP 2008. Lecture Notes in Computer Science, v.5425, pp.112-120, 2008. 
[4] G.T. Amariucai and S. Wei, "Jamming in Fixed-Rate Wireless Systems with Power Constraints - Part I: Fast Fading Channels", arXiv:0808.3431v1 [cs.IT], 2008.

[5] G.T. Amariucai, S. Wei and R. Kannan, "Jamming in Fixed-Rate Wireless Systems with Power Constraints - Part II: Parallel Slow Fading Channels". arXiv:0808.3418v1 [cs.IT], 2008.

[6] T. Basar, "The Gaussian test channel with an intelligent jammer," IEEE Trans. Inform. Theory, vol. 29, pp. 152157, 1983.

[7] A. Garnaev, Search Games and Other Applications of Game Theory, Springer, 2000.

[8] S.Hart, "Discrete Colonel Blotto and General Lotto games," Int J Game Theory, vol. 36, pp. 441460, 2008.

[9] H. Holma and A. Toskala. WCDMA for UMTS

[10] H. Ji and C.-Y. Huang, "Non-cooperative uplink power control in cellular radio systems", Wireless Networks, v.4, pp.233-240, 1998.

[11] S. L. Kim, Z. Rosberg and J. Zander. Combined power control and transmission selection in cellular networks. In Proceedings of IEEE Vehicular Technology Conference, Fall 1999.

[12] I. Koo, J. Ahn, H. A. Lee, and K. Kim, Analysis of Erlang capacity for the multimedia DS-CDMA systems. IEICE Trans. Fundamentals, E82-A(5):849-855, May 1999.

[13] M. Medard, Capacity of correlated jamming channels, Allerton Annual Conf. on Comm., Control and Computing, 1997.

[14] S. Ray, P. Moulin, M. Medard, On Optimal Signaling and Jamming Strategies in Wideband Fading Channels, IEEE Workshop on Signal Processing Advances in Wireless Communications (SPAWC) 2006, Cannes, France, July 2006.

[15] S. Shafiee and S. Ulukus, Correlated jamming in multiple access channels, Conference on Information Sciences and Systems, March 2005.

[16] D. Tse and P. Viswanath, Fundamentals of Wireless Communication, Cambridge University Press, 2005. 